\title{
Moluscos de la expedición del R/V Edwin Link en las costas del Caribe mexicano
}

\author{
Norma Emilia González ${ }^{1}$ \\ Depto. Ecología Acuática, El Colegio de la Frontera Sur, Apdo. Postal 424, Chetumal, Q.R. 77000 México, emilia@eco- \\ sur-qroo.mx
}

Recibido 7-VIII-1997. Corregido 16-VI-1998. Aceptado 23-VI-1998.

\begin{abstract}
A checklist of marine mollusc species mainly from deep-water localities off Mexican Caribbean shores is presented. Samples were obtained with a mechanical arm in 2-307 m during the expedition of the R/V "Edwin Link", August 1990; the list includes 59 species of Gastropoda, 17 species of Bivalvia, three species of Scaphopoda and three species of Polyplacophora.
\end{abstract}

Key words: Mollusca, deep-water, Caribbean Sea, checklist.

La mayoría de los estudios de moluscos que se han realizado en el litoral del Caribe mexicano tratan sobre sistemática, aspectos ecológicos y distribución. De los más recientes en la Península de Yucatán destacan los de Vokes y Vokes (1983), Bolívar de Carranza e Hidalgo-Escalante (1990), González et al. (1991); todos versan sobre organismos de aguas poco profundas y de la zona arrecifal. Sobre fauna de aguas profundas están los que realizaron Dall (1889) en el golfo de México y mar Caribe, Rehder y Abbott (1951) en el golfo de México y posteriormente Rice y Kornicker (1965) para la sonda de Campeche. Ekdale $(1974,1978)$ recolectó con una bomba de succión en fondos a 20-60 m, en la costa noreste entre Isla Contoy, Isla Mujeres y Cancún. Treece (1980) recolectó en 11 sitios, en la punta nornoreste de la península a una profundidad de 5-80 $\mathrm{m}$.

Este trabajo presenta una lista de los moluscos recolectados con el brazo mecánico del submari- no del buque "Edwin Link", e incluye los sitios y profundidad de muestreo de cada especie. Se guardó un registro fotográfico por especie que se depositó junto con el material en la Colección de Referencia del Laboratorio de Bentos de El Colegio de la Frontera Sur (QNR.IN.021.0497).

Gran parte de las muestras corresponden a Banco Chinchorro, atolón de $46 \mathrm{~km}$ de largo y 14 $\mathrm{km}$ de ancho. Pertenece al gran cinturón de arrecifes del Atlántico y está separado del continente por un canal de $1000 \mathrm{~m}$ de profundidad; tiene forma semioval y presenta cuatro cayos arenosos. Cayo Norte fue el sitio del que se revisaron más muestras, pero todas contenían de 1 a 6 organismos como máximo. La recolecta de los organismos fue en agosto de 1990 y fueron proporcionadas por Elva Escobar y Luis Soto del Instituto de Ciencias del Mar y Limnología y por José Luis Villalobos del Instituto de Biología ambos de la Universidad Nacional Autónoma de México (Apéndice 1). 
Para la identificación taxonómica se utilizaron las obras de Warmke y Abbott (1962), Abbott (1974), Morris (1975), Rehder (1981), Vokes y Vokes (1983), Díaz-Merlano y Puyana-Hegedus (1994) y se compararon con los especimenes de la colección de referencia mencionada. El arreglo sistemático de las especies (Cuadro 1), se hizo siguiendo el criterio de Abbott (1974), y para la actualización de las familias de gasterópodos se consultó la base de datos elaborada por Rosenberg (1993) y disponible en internet (gopher://erato.acnatsci.org.).
Se procesaron 244 organismos, el $64 \%$ de los moluscos estaban vivos y el resto de las conchas estaban ocupadas por cangrejos ermitaños o vacías. Se identificaron 82 especies, la mayoría micromoluscos, que pertenecen a las siguientes clases, indicándose en paréntesis el número de especies para cada uno: Gastropoda (59), Bivalvia (17), Scaphopoda (3), Polyplacophora (3). La familia mejor representada fue Turbinidae con 16 especies.

CUADRO 1

Lista sistemática de las especies de moluscos recogidos por el $R V$ "Edwin Link" en el Caribe mexicano.

\author{
Taxon \\ Clase Gastropoda \\ Orden Archaeogastropoda \\ Familia Haliotidae \\ Haliotis pourtalesii Dall 1881 \\ Familia Fissureliidae \\ Lucapina sowerbi (Sowerby 1835) \\ Familia Trochidae \\ Calliostoma barbouri (Clench \& Aguayo 1946)
}

Solariella lacunella (Dall 1881)

Tegula fasciata (Born 1778)

Familia Liotiidae

Arene bairdii (Dall 1889)

Arene briareus (Dall 1889)

Familia Turbinidae

Astraea tecta americana (Gmelin 1791)

Astraea phoebia (Röding 1798)

Astraea caelata (Gmelin 1791)

Turbo castanea Gmelin 1791

Turbo haraldi Robertson 1957

Turbo cailletii Frischer y Bernardi 1856

Tricolia bella (M. Smith 1937)

Tricolia thalassicola Robertson 1958

Tricolia affinis (C.B. Adams 1850)

Familia Turritellidae

Turritella exoleta (Linné 1758)

Vermicularia knorri (Deshayes 1843)

Familia Turridae

Drillia cydia Bartsch 1943

Drilla albicoma (Dall 1889)

Splendrilla moseri (Dall 1889)
Estación/profundidad

$2793 / 117 m$

$2782 / 274 m$

$2774 / 60 \mathrm{~m}$

$2783 / 241 \mathrm{~m}$

$2278 / 204 \mathrm{~m}$

$2788 / 243 \mathrm{~m}$

$2791 / 175 m$

$2793 / 116 m$

$2783 / 60 \mathrm{~m}$

$2777 / 78 \mathrm{~m}$

$2786 / 244 \mathrm{~m}$

$2777 / 78 \mathrm{~m}$

$2792 / 158 \mathrm{~m}$

$2778 / 203 \mathrm{~m}$

2785/20m

$2785 / 244 \mathrm{~m}$

$2783 / 60 \mathrm{~m}$

$2780 / 267 \mathrm{~m}$

$2775 / 50 \mathrm{~m}$

$2788 / 243 m$

$2775 / 50 \mathrm{~m}$

$2782 / 257 \mathrm{~m}$

2778/200m 
Familia Siliquariidae

Tenagodus squamatus Blainville 1827

$2783 / 240 \mathrm{~m}$

Tenagodus modestus Dall 188

$2789 / 78 \mathrm{~m}$

Familia Vermetidae

Dendropoma irregulare (Orbigny 1842)

$2780 / 4 m$

Familia Cerithidae

Cerithium litteratum (Born 1758)

$2779 / 4 m$

Bittium varium (Pfeiffer 1840)

$2779 / 4 \mathrm{~m}$

Familia Triphoridae

Isotriphora peetersae (Moolenbeck \& Faber 1989)

$2783 / 58 m$

Familia Triviidae

Trivia quadripunctata (Gray 1827)

$2782 / 5 \mathrm{~m}$

Trivia antillarum Schilder 1922

$2793 / 116 m$

Familia Atlantidae

Atlanta peroni Lessueur 1827

$2782 / 225 \mathrm{~m}$

Atlanta inclinata Gray 1850

$2782 / 225 m$

Familia Ovulidae

Cyphoma gibbosum Linné 1881

$2793 / 116 \mathrm{~m}$

$2782 / 5 \mathrm{~m}$

Familia Ranellidae

Cymatium moritinctum Clench \& Turner 1957

$2788 / 112 m$

Orden Neogastropoda

Familia Muricidae

Murex pomum Gmelin 1791

$2779 / 4 m$

Murexiella macgintyi M. Smith 1938

$2782 / 6 \mathrm{~m}$

Familia Columbellidae

Costoanachis lafresnayi (Fischer \& Bernardi 1856)

$2783 / 240 \mathrm{~m}$

Costoanachis sparsa (Reeve 1859)

$2777 / 66 \mathrm{~m}$

Columbella mercatoria (Linné 1758)

$2782 / 91 \mathrm{~m}$

Familia Buccinidae

Engina turbinella (Kiener 1835)

$2779 / 78 m$

Familia Nassariidae

Nassarius albus (Say 1826)

$2788 / 95 m$

Familia Fasciolariidae

Fasciolaria lilium Fischer 1807

Familia Volutomitridae

Conomitra cf. lindae Petuch 1987

$2777 / 236 m$

Familia Cystiscidae

Persicula fluctuata (CB Adams 1850)

$2772 / 69 m$

Familia Marginellidae

Volvarina albolineata (Orbigny 1842)

$2780 / 78 \mathrm{~m}$

Volvarina avena (Kiener 1834)

$2777 / 5 \mathrm{~m}$

Volvarina veilei (Kiener 1841)

$2789 / 307 \mathrm{~m}$ 
Hyalina lactea (Kiener 1841)

2793/116m

Prunum amabile (Redfield 1852)

$2791 / 175 \mathrm{~m}$

Prunum labiatum (Kiener 1841)

$2782 / 6 m$

Familia Conidae

Conus jaspideus Gmelin 1791

$2779 / 4 \mathrm{~m}$

Conus floridanus Gabb 1868

$2783 / 60 \mathrm{~m}$

Conus daucus Hwass 1792

$2788 / 243 m$

Familia Bullidae

Atys sandersoni Dall 1881

$2775 / 212 \mathrm{~m}$

Bulla striata Bruguière 1792

$2793 / 116 m$

Suborden Thecosomata

Familia Cavoliniidae

Diacavolinia longirostris (Blainville 1821)

Cavolinia trispinosa (Blainville 1821)

$2789 / 307 \mathrm{~m}$

$2774 / 51 \mathrm{~m}$

$2775 / 50 \mathrm{~m}$

Cavolinia uncinata (Range 1829)

$2782 / 225 m$

Clase Scaphopoda

Familia Dentaliidae

Dentalium semistriolatum Guilding 1834

$2779 / 78 \mathrm{~m}$

$2775 / 212 \mathrm{~m}$

Dentalium pilsbryi Rehder 1942

Dentalium ceratum Dall 1881

$2778 / 209 m$

Clase Amphineura

Orden Chitonida

Familia Ischnochitonidae

Stenoplax floridana (Pilsbry 1892)

$2788 / 243 m$

$2780 / 266 \mathrm{~m}$

Familia Acantochitonidae

Cryptoconchus floridanus (Dall 1889)

$2792 / 2 m$

Clase Bivalvia

Orden Arcoida

Familia Arcidae

Barbatia cancellaria (Lamarck 1819)

Barbatia dominguensis (Lamarck 1819)

$2779 / 78 \mathrm{~m}$

$2774 / 51 \mathrm{~m}$

Arcopsis adamsi ( Dall 1886)

$2774 / 51 \mathrm{~m}$

Familia Glycymerididae

Glycymeris undata (Linné 1758)

$2774 / 51 \mathrm{~m}$

Glycymeris pectinata (Gmelin 1791)

$2774 / 60 \mathrm{~m}$

Superfamilia Pectinacea

Familia Pectinidae

Chlamys nana (Verrill \& Bush 1897)

Familia Plicatulidae

Plicatula gibbosa Lamarck 1801

Familia Limidae

Lima lima (Linné 1758)

Lima pellucida CB Adams 1846

Lima scabra tenera (Bom 1778)

$2780 / 266 m$

$2782 / 91 \mathrm{~m}$

$2781 / 60 \mathrm{~m}$

$2781 / 60 \mathrm{~m}$

$2788 / 243 m$ 


\author{
Suclase Heterodonta \\ Orden Veneroida \\ Familia Ungulinidae \\ Diplodonta punctata (Say 1822) \\ Superfamilia Chamacea \\ Familia Chamidae \\ Chama congregata \\ Familia $\mathrm{C}$ rditid e \\ Glans dominguensis (Orbygny 1845) \\ Familia Cardiidae \\ Laevicardium laevigatum (Linné 1758) \\ Familia Veneridae \\ Chione cancellata (Linné 1767) \\ Pitar fulminata (Menke 1828) \\ Orden Myoida \\ Superfamilia Myacea \\ Familia Corbulidae \\ Corbula dietziana CB Adams 1852
}

$2779 / 78 \mathrm{~m}$

$2779 / 78 \mathrm{~m}$

$2774 / 51 \mathrm{~m}$

$2785 / 244 m$

$2774 / 51 \mathrm{~m}$

$2774 / 51 \mathrm{~m}$

$2793 / 116 m$

Nota: Tamaño de muestra, 1-10 ejemplares (mayoría: 1-3).

Nuevos registros y observaciones.

Haliotis pourtalesii (Dall 1881)

Registros previos: Titgen y Brigth (1985) a $\operatorname{los} 21^{\circ} 21^{\prime} \mathrm{N}$ y $86^{\circ} 30^{\prime} \mathrm{W}$, Noreste de la Península de Yucatán una concha vacía a $123 \mathrm{~m}$ de profundidad.

Material examinado: Seis ejemplares recolectados vivos, a los $21^{\circ} 16.6^{\prime} \mathrm{N}$ y $86^{\circ} 38.5^{\prime} \mathrm{W}$, a 12 kilometros de la punta norte de Isla Mujeres, Q.R. a $117 \mathrm{~m}$ de profundidad.

Observaciones: Es el primer registro de organismos vivos para el Caribe mexicano. La longitud promedio de las conchas fue de $19.1 \mathrm{~mm}$, sólo uno midió $10.4 \mathrm{~mm}$. Al separar la concha de un organismo se notaron las gónadas de gran tamaño, es probable que cuando se recolectó estaban en época reproductiva. 1946)

\section{Calliostoma barbouri (Clench y Aguayo}

Registros previos: Aguas poco profundas de Cuba y Colombia.

Material examinado: Cinco ejemplares recolectados, sólo uno de ellos vivo, frente a Ma- hahual $\left(18^{\circ} 41.85^{\prime} \mathrm{N}\right.$ y $\left.87^{\circ} 41.58^{\prime} \mathrm{W}\right)$ y en Banco Chinchorro $\left(18^{\circ} 45.63^{\prime} \mathrm{N}\right.$ y $87^{\circ} 15.84^{\prime} \mathrm{W}$ ) a $60 \mathrm{~m}$ y $241 \mathrm{~m}$ respectivamente.

Observaciones: El tamaño promedio de los organismos fue $12 \mathrm{~mm}$. Quinn (1992) mencionó que la profundidad de esta especies es de 25$100 \mathrm{~m}$, para la zona del Caribe mexicano se ampliaría a un ámbito de $241 \mathrm{~m}$.

Turbo haraldi (Robertson 1957)

Registros previos: Bahamas, Dominicana, Barbados y Panamá.

Material examinado: 6 ejemplares recolectados vivos, al sur de Cayo Blackford (Banco Chinchorro) a $18^{\circ} 24.64^{\prime} \mathrm{N}$ y $87^{\circ} 25.6^{\prime} \mathrm{W}$, en profundidades de 66 y $203 \mathrm{~m}$.

Observaciones: Tenían los organismos una longitud promedio de $12 \mathrm{~mm}, 4$ ejemplares eran totalmente anaranjados con la protoconcha en tono rosado y sólo uno presentaba la coloración de la descripción original de Robertson. La profundidad previamente registrada era de 50-68m, con este registro se amplía hasta $203 \mathrm{~m}$. 
Isotriphora peetersae (Moolenbeck \& Faber 1989)

Registros previos: Aruba y Cuba

Material examinado: Una concha vacía de $7 \mathrm{~mm}$ de largo. Localizada frente a Mahahual, Q.R. a $18^{\circ} 41.8^{\prime} \mathrm{N}$ y $87^{\circ} 41.8^{\prime} \mathrm{W}$, recolectada a $58 \mathrm{~m}$

Observaciones:Moolenbeck y Faber (1989) describen a $I$. peetesae como una especie con una típica protoconcha blanca paucispiral; fué la primera para aguas caribeñas. Mas tarde, Rolán y Espinosa (1994), refieren que ellos encontraron varios especimenes con la protoconcha nodular blanca, y sugieren que existe un complejo de especies, porque notaron otras diferencias en el patrón de coloración y en la rádula, el organismo aquí señalado es muy similar al encontrado en aguas cubanas.

Conomitra cf. lindae (Petuch 1987)

Registros previos: Caribe colombiano.

Material examinado: Dos ejemplares, ambos las conchas vacías, medían un promedio de $8 \mathrm{~mm}$ de longitud. Fueron recolectados al sur de Banco Chinchorro en la zona expuesta $\left(18^{\circ}\right.$ $26.02^{\prime} \mathrm{N}$ y $87^{\circ} 18.82^{\prime} \mathrm{W}$ ).

Observaciones: Comparte algunas características de $C$. blakeana,( Dall 1889) reportada para el Canal de Yucatán, a 640 brazas, y menciona que presenta color ciruela cuando fresco, y café pardo con blanco cuando está deteriorada. Igual que en este trabajo, sólo dos especímenes se colectaron. Mientras que con $C$. lindae (Petuch 1987) comparte la ornamentación de finas costillas axiales intersectadas por costillas espirales, formando pequeños nudos y la columnela con 4 pliegues, color crema con manchas marrón; la protoconcha difiere, es café para $C$. lindae, y en los organismos reportados aquí es color blanca.

Las condiciones ecológicas que presenta el Banco Chinchorro, como fondos rocosos, altas temperaturas y transparencia del agua, podría explicar el cambio en la batimería de las especies que normalmente se ubican en aguas poco profundas. La explicación para las especies que no habían sido encontradas para esta zona podría deberse a la falta de estudios previos.

\section{REFERENCIAS}

Abbott, R.T. 1974. American Seashells: The marine Mollusca of the Atlantic and Pacific coast of North America. van Nostrand Reinhold, Nueva York, 663 p.

Bolívar de Carranza, A.M. \& E. Hidalgo-Escalante. 1990. Lista de moluscos gasterópodos y pelecípodos del golfo de México y Mar Caribe. An. Esc. Nac. Cienc. Biol. IPN México 33:53-72.

Dall, W.H. 1889. Report on the results of dredging, under the supervision of Alexander Agassiz, in the Gulf of Mexico (1877-78) and in the Caribbean Sea (1879-80) by the US coast survey streamer "Blake", Part II. Gastropoda and Scaphopoda. Bull. Mus. Comp. Zool. Harvard 18:1-492.

Díaz-Merlano, J.M. \& M. Hegedus-Puyana. 1994. Moluscos del Caribe Colombiano: Un catálogo ilustrado. Colciencia, Fund. Nat e Invermar. Santafe de Bogotá, 291 p.

Ekdale, A.A. 1974. Marine molluscs from shallow-water enviroments ( 0 a 60 meters) off the Northeast Yucatan coast, Mexico. Bull .Mar. Sci. 24:638-667.

Ekdale, A.A. 1978. Recent marine molluscs from northeastern Quintana Roo. Mexico 113-133 pp In: W.C. Ward \& A. E. Weidie (eds.) Geology and Hydrogeology of Northeastern Yucatán. New Orleans Geol. Soc. Nuevo Orleans, 327p.

González, M.A., E. Chávez, G. de la Cruz \& D. Torruco. 1991. Patrones de distribución de gasterópodos y bivalvos en la Península de Yucatán, Méx. Cienc. Mar. 17:147-172.

Morris, P.A. 1975. A Field Guide to Shells of the Atlantic and Gulf coast and the West Indies. Houghton Mifflin, Boston 330p.

Quinn, J.F. 1992. New species of Calliostoma Sawinson, 1840 (Gastropoda: Trochidae), and notes on some poorly known species from the Western Atlantic Ocean. Nautilus 106:77-114.

Rehder, H.A. \& R.T. Abbott 1951. Some new and intersting mollusks from the deeper waters of the Gulf of Mexico. Rev. Soc. Malacol. "Carlos de la Torre", La Habana, Cuba 8:53-66.

Rehder, H.A. 1981. The Audobon Society Field Guide to North American Seashells. Chanticleer, Nueva York, 894 p. 
Rice, W.H. \& L. S. Kornicker. 1965. Mollusks from the deeper waters of the northwestern Campeche Bank, Mexico. Publ. Inst. Mar. Sci. Univ. Texas 10:108-172.

Rolán, E. \& J. Espinosa. 1994. The family Triphoridae (Mollusca, Gastropoda Prosobranchia) in Cuba, 3. The genus Isotriphora, with description of a new species. Basteria 58:63-68.

Rosenberg, G. 1993. A data base approach to studies of molluscan taxonomy, biogeography and diversity, with examples from western Atlantic marine gastropods. Am. Mal. Bull. 10:257-266.
Titgen, R.H. \& T.J. Bright.1985. Notes on the distribution and ecology of the western Atlantic abalone, Haliotis pourtalesii Dall 1881 (Mollusca:Gastropoda). Northeast Gulf Sci. 7:147-152.

Treece, G.D. 1980. Bathymetric records of marine shelled mollusca from the northeastern shelf and upper slope of Yucatan, Mexico. Bull. Mar. Sci. 30:552-570.

Vokes, H.E. \& E.H. Vokes. 1983. Distribution of shallow water marine Mollusca of the Yucatan Peninsula, Mexico. Tulane Univer., Nueva Orleans 183 p.

Warmke, G.L. \& R.T. Abbott. 1962. Caribbean Seashells. Dover, Nueva York, 348 p.

\section{APENDICE 1}

Sitios de recolección con la respectiva clave para cada estación.

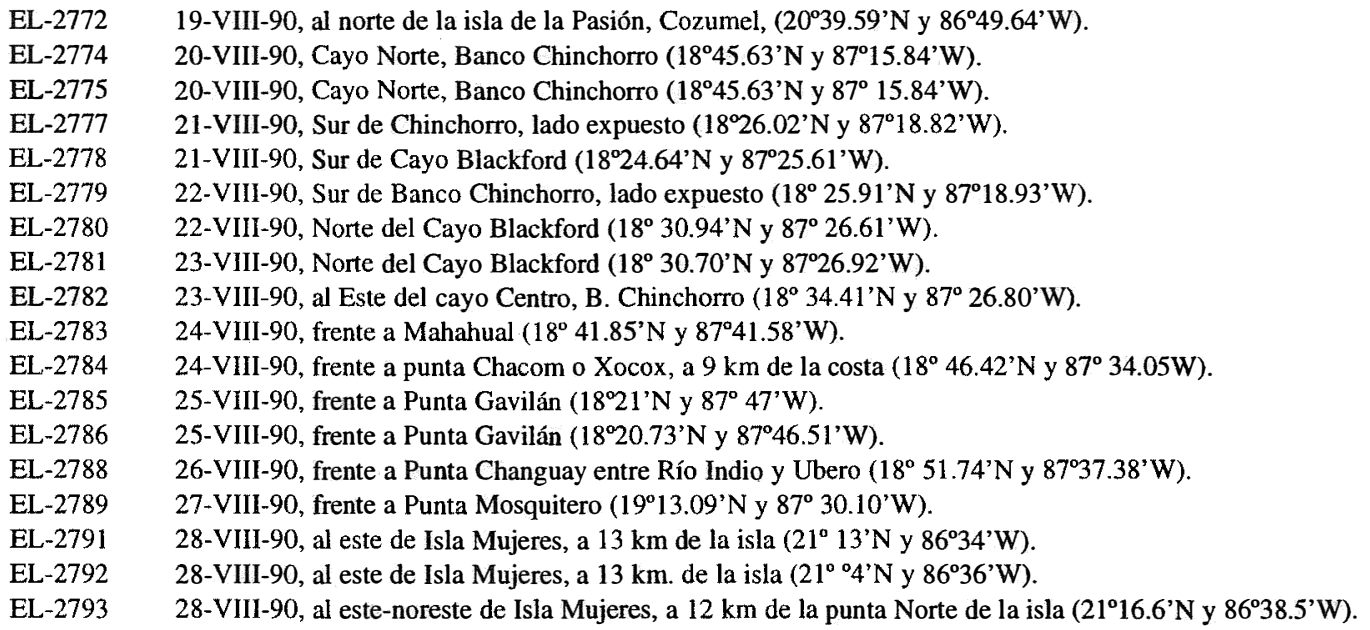

